\title{
Generation of correlated biphoton via four-wave mixing coexisting with multi-order fluorescence
} processes

\author{
Yang Liu ${ }^{1,2,3}$, Kangkang Li ${ }^{1}$, Siqiang Zhang ${ }^{1}$, Huanrong $\mathrm{Fan}^{1}{ }^{1}$, Wei $\mathrm{Li}^{1}$ \& Yanpeng Zhang ${ }^{1 *}$ \\ We investigate the parametrically amplified four-wave mixing, spontaneous parametric four- \\ wave-mixing, second- and fourth-order fluorescence signals coming from the four-level double- $\boldsymbol{\Lambda}$ \\ electromagnetically induced transparency system of a hot ${ }^{85} \mathrm{Rb}$ atomic vapor. The biphoton temporal \\ correlation is obtained from spontaneous parametric four-wave-mixing and fourth-order fluorescence \\ processes. Meanwhile, we first observed the biphoton Rabi oscillation with a background of linear \\ Rayleigh scattering and uncorrelated second-order fluorescence. The outcomes of the investigation \\ may contribute potentially to the applications in dense coding quantum communication systems.
}

In recent five decades, the generation of time-energy entangled photon pairs has attracted worldwide attention, because these correlations are central to the foundational questions in quantum mechanics ${ }^{1}$ and play a vital role in application oriented research of quantum communication ${ }^{2}$, computation $^{3}$, quantum imaging ${ }^{4,5}$, and quantum metrology $y^{6,7}$. Generally, the correlated photon pairs are generated by spontaneous parametric down-conversion (SPDC) in nonlinear crystals ${ }^{8,9}$. However, the photon pairs from this nonlinear process usually have wide bandwidth $(\mathrm{THz})$, short coherence time $(\mathrm{ps})$ and short coherence length $(100 \mu \mathrm{m})$, which comes as a limitation for long-distance fiber optical quantum communication ${ }^{10}$. To solve this problem, Du's group generated subnatural-linewidth correlated biphoton from the spontaneous parametric four-wave mixing (SP-FWM) in the cold atoms $(10-100 \mu \mathrm{K})^{11-14}$. SP-FWM nonlinear process can produce narrow-band (MHz) and ultra-long coherence time $(\mu \mathrm{s})$ two-mode entanglement source. Moreover, an SP-FWM process can generate correlated photon pairs of Stokes $\left(E_{S}\right)$ and anti-Stokes $\left(E_{a S}\right)$ coexisting with multi-order fluorescence $(F L)$ and Rayleigh scattering signals simultaneously. In addition, $E_{S}$ and $E_{a S}$ can also be used in an optical parametric amplification (OPA) process to research the squeezed and entangled states of optical fields $s^{15-20}$. Currently, a great deal of work has been done in studying the mechanism of the nonlinear optical process, such as the influence of dressing fields on parametric amplification of four-wave mixing (PA-FWM) processes in a "double- $\Lambda$ " atomic system ${ }^{21}$.

In this paper, we propose the experimental demonstration of the generation of narrow-bandwidth nondegenerate paired photons from a hot ${ }^{85} \mathrm{Rb}$ atomic ensemble via coexisting SP-FWM and multi-fluorescence. We observed the biphoton Rabi oscillations with a background of linear Rayleigh scattering and second-order fluorescence. These nonlinear optical processes are controlled by adjustable detuning of pump and coupling fields. The outcomes of the investigation may potentially contribute to the applications in dense coding quantum communication systems. The paper is constructed as follows: in section II, firstly we study the generation processes of PA-FWM, SP-FWM and multi-order fluorescence. Then calculate the coincidence counting of the SP-FWM and correlation of multi-order fluorescence. In section III, we study the influence between the detuning of pump field and EIT windows. Then discuss the biphoton correlations of SP-FWM and multi-order fluorescence. In section IV, we conclude the paper.

\footnotetext{
${ }^{1}$ Key Laboratory for Physical Electronics and Devices of the Ministry of Education \& Shaanxi Key Laboratory of Information Photonic Technique, Xi'an Jiaotong University, Xi'an, 710049, China. ${ }^{2}$ State Key Laboratory of Transient Optics and Photonics, Xi'an Institute of Optics and Precision Mechanics, Chinese Academy of Sciences, Xi'an, 710119, China. ${ }^{3}$ University of Chinese Academy of Sciences, Beijing, 100049, China. *email: ypzhang@mail.xjtu.edu.cn
} 


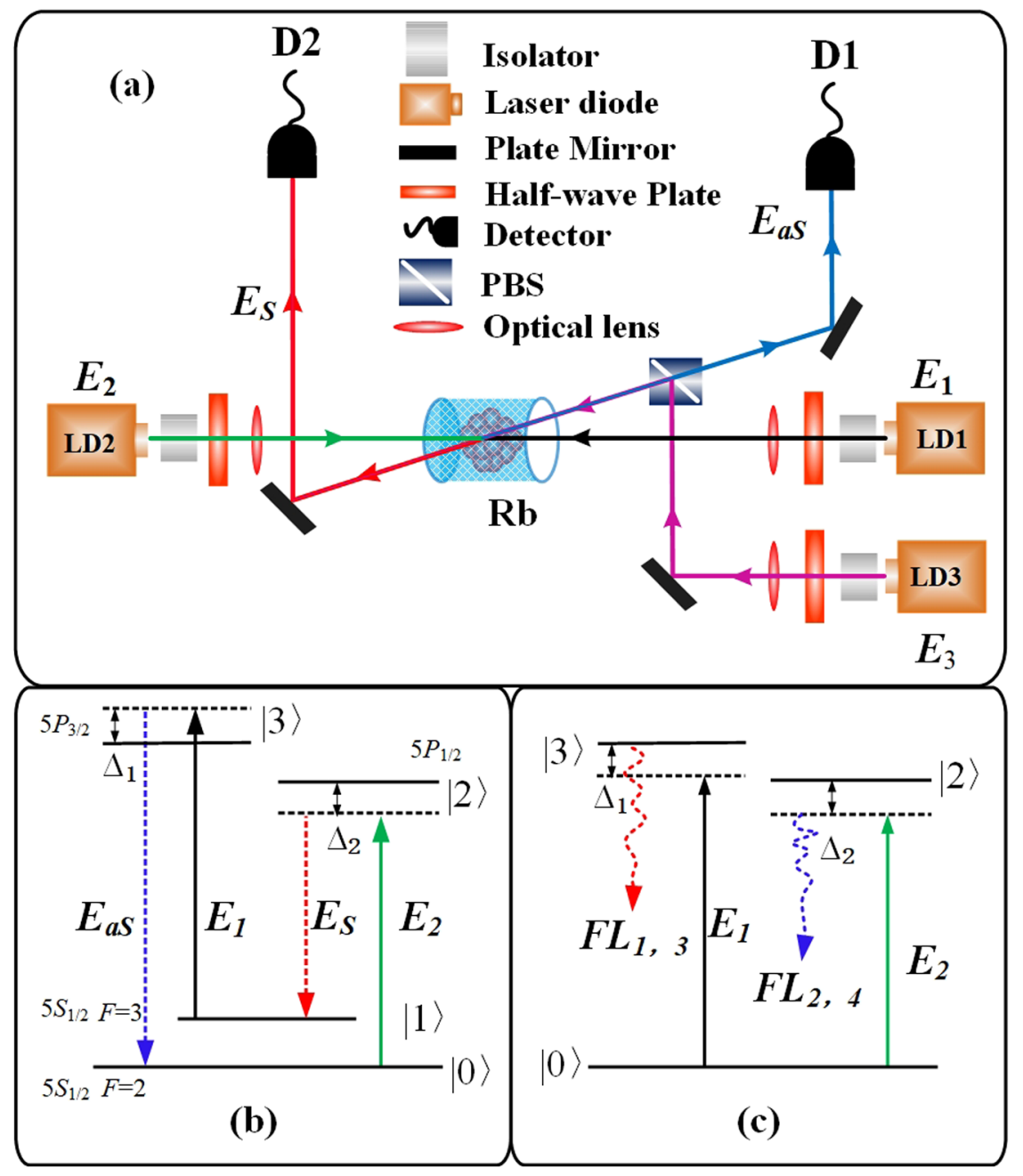

Figure 1. (a) The experimental setup and spatial beams alignment of the FWM and fluorescence processes; I: isolator; LD1-3: laser diode; PBS: polarization beam splitter; D1-2: avalanche photodiode detector (APD) or single-photon counting module (SPCM). (b) The energy diagrams of FWM generation processes in "double- $\Lambda$ " four-level atomic systems of ${ }^{85} \mathrm{Rb}$, respectively. $E_{s}$ and $E_{a S}$ denote the Stokes and anti-Stokes signals. (c) The energy diagrams of fluorescence generation processes. $F L_{1,2}$ and $F L_{3,4}$ denote the second- and fourth-order fluorescence signals.

\section{Basic Theory}

We start the experiment description with the spatial beams alignment and associated energy level diagram of the FWM and fluorescence processes shown in Fig. 1. The experiments are carried out in a "double- $\Lambda$ " four-level atomic systems of ${ }^{85} \mathrm{Rb}$ shown in Fig. 1(b). The $|0\rangle\left(5 S_{1 / 2}, \mathrm{~F}=2\right),|1\rangle\left(5 S_{1 / 2}, \mathrm{~F}=3\right),|2\rangle\left(5 P_{1 / 2}\right)$ and $|3\rangle\left(5 P_{3 / 2}\right)$ are four relevant atomic energy levels. The strong pump laser beam $E_{1}$ (frequency $\omega_{1}$, wave vector $\mathbf{k}_{1}$, Rabi frequency $G_{1}$, wavelength $780 \mathrm{~nm}$, power up to $\left.54.5 \mathrm{~mW}\right)$ connecting $|0\rangle\left(5 S_{1 / 2}, \mathrm{~F}=3\right)$ and $|3\rangle\left(5 P_{3 / 2}\right)$ comes from the laser diode 1 (LD1). The coupling laser beam $E_{2}\left(\omega_{2}, \mathbf{k}_{2}, G_{2}, 795 \mathrm{~nm}, 39 \mathrm{~mW}\right)$ connecting $|1\rangle\left(5 S_{1 / 2}, \mathrm{~F}=2\right)$ and $|2\rangle\left(5 P_{1 / 2}\right)$ is emitted by the LD2. The weak probe laser beam $E_{3}\left(\omega_{3}, \mathbf{k}_{3}, G_{3}, 780 \mathrm{~nm}, 7.2 \mathrm{~mW}\right)$ connecting $|3\rangle\left(5 P_{3 / 2}\right)$ and $|1\rangle$ $\left(5 S_{1 / 2}, \mathrm{~F}=3\right)$ comes from the LD3 in the $E_{1}$ direction. As indicated in Fig. 1(a), the incident beam $E_{1}$ propagates in the same direction with $E_{3}$, and can form a standard $\Lambda$-EIT window, while the $E_{1}$ propagates in the opposite direction with $E_{2}$, which also generate a new type EIT window. The FWM signals and transmitted probe beam in Fig. 1(c) are detected by an avalanche photodiode detector (APD) and satisfy the phase matching condition (PMC) of $\mathbf{k}_{a S}=\mathbf{k}_{1}+\mathbf{k}_{2}-\mathbf{k}_{S}$. In addition, if we block the injection laser $E_{3}$ and change the detectors for two single-photon counting modules (SPCM), the biphoton coincidence counts with fluorescence signals can be detected.

\section{Generation Process of SP-FWM and Coincidence Counting}

Firstly, we block the injection laser $E_{3}$ to get the SP-FWM $\left(E_{S}\right.$ and $\left.E_{a S}\right)$ which can be described by the third-order density matrix elements (the solutions are given in the Methods). The phase matching condition $\left(\mathbf{k}_{1}+\mathbf{k}_{2}=\mathbf{k}_{S}+\mathbf{k}_{a S}\right)$ of the two spontaneous emission signals is satisfied. Generally, we assume that the $E_{S}$ and $E_{a S}$ fields are much weaker than the $E_{1}$ and $E_{2}$, so they are regarded as two classical fields $\left(E_{1}\right.$ and $\left.E_{2}\right)$ and two 
quantum fields (described as $a^{\dagger}$ and $b^{\dagger}$ ) with the Hamiltonian $H=i \hbar \kappa \hat{a}^{\dagger} \hat{b}^{\dagger}+h$.c., respectively. Where $\kappa$ is the nonlinear coefficient and can be expressed as $\kappa=\left|\chi_{S / a S}^{(3)} E_{1} E_{2}\right|=\left|N \mu_{10}^{2} \rho_{S / a S}^{(3)} / \hbar \varepsilon_{0} G_{S / a S}\right|$. According to the perturbation theory, it can be described as the models of the $E_{S}$ and $E_{a S}$, the correspondings perturbation chains are $\rho_{11}^{(0)} \stackrel{\omega_{1}}{\rightarrow} \rho_{31}^{(1)} \stackrel{-\omega_{a S}}{\longrightarrow} \rho_{01}^{(2)} \stackrel{\omega_{2}}{\rightarrow} \rho_{21(S)}^{(3)}$ and $\rho_{00}^{(0)} \stackrel{\omega_{2}}{\rightarrow} \rho_{20}^{(1)} \stackrel{-\omega_{S}}{\longrightarrow} \rho_{10}^{(2)} \stackrel{\omega_{1}}{\rightarrow} \rho_{30(a S)}^{(3)}$ respectively.

The propagation equation of Stokes and anti-Stokes photons can be written as: $\frac{d}{d t} \hat{a}=k \hat{b}^{\dagger}$ and $\frac{d}{d t} \hat{b}=k \hat{a}^{\dagger}$. where $a^{\dagger}$ and $b^{\dagger}$ are creation operators for Stokes and anti-Stokes photons, respectively. Solving these above two equations, we can obtain the output Stokes and anti-Stokes fields as: $\hat{a}(t)=\cosh (k t) \hat{a}(0)+\sinh (k t) \hat{b}^{\dagger}(0)$ and $\hat{b}^{\dagger}(t)=\sinh (k t) \hat{a}(0)+\cosh (k t) \hat{b}^{\dagger}(0)$. The interaction of Hamiltonian determines the evolution of the two-photon state vector ${ }^{22}$. The mechanism of biphotons generation near the resonance is explained clearly ${ }^{23}$. The biphoton amplitude in the time domain can be expressed as:

$$
\psi(\tau)=\frac{L}{2 \pi} \int d \omega_{a s} \kappa\left(\omega_{a s}\right) \Phi\left(\omega_{a s}\right) e^{-i \omega_{a s} \tau} .
$$

where $\Phi\left(\omega_{a s}\right)$ is the longitudinal detuning function, and can be written as $\Phi\left(\omega_{a s}\right)=\operatorname{sinc}\left(\frac{\Delta k L}{2}\right) e^{i \frac{L}{2}\left[k_{s}\left(\omega_{a s}\right)+k_{a s}\left(\omega_{a s}\right)\right]}$, and the relative time delay $\tau=t_{a S}-t_{s}$. The biphotons wave function is determined by both the longitudinal detuning function and nonlinear coupling coefficient. The third-order nonlinear susceptibility of the anti-Stokes field can be defined as:

$$
\chi_{a S}^{(3)}=\frac{N \mu_{13} \mu_{14} \mu_{23} \mu_{24}}{\varepsilon_{0} \hbar^{3}} \frac{1}{\left(\Gamma_{20}+\mathrm{i} \Delta_{2}\right)\left(\Gamma_{10}+\mathrm{i} \delta\right)\left(\Gamma_{30}+\mathrm{i} \delta+\mathrm{i} \Delta_{1}\right)} .
$$

where $\mu_{i j}$ are the electric dipole matrix elements and $\Gamma_{i j}$ are the dephasing rates of coherence $|i\rangle \rightarrow|j\rangle$. $\Delta_{1}=\omega_{31}-\omega_{1}$ and $\Delta_{2}=\omega_{20}-\omega_{2}$ are the detunings of coupling and pump field. The damped oscillation with a frequency of $\Delta_{1}$ results from the interference between two resonance bands at $\delta=0$ and $\Delta_{1}$. The two-photon coincidence counting rate can be calculated as:

$$
\operatorname{Rcc}(\tau)=W\left[1-\cos \left(\Delta_{1} \tau\right)\right] e^{-2 \Gamma_{e} \tau} .
$$

where $W$ is a constant, $\tau_{r}=2 \pi / \Delta_{1}$ is the Rabi period, $\Gamma_{e}=\left(\Gamma_{10}+\Gamma_{30}\right) / 2$ is the effective dephasing rate and $\tau_{e}=\Gamma_{e} / 2$ is the nonlinear coherence time.

\section{Generation and Correlation of Multi-Order Fluorescence}

In the system, the second-order fluorescence is generated through the perturbation chains $\rho_{00}^{(0)} \stackrel{G_{1}}{\rightarrow} \rho_{30}^{(1)} \stackrel{G_{1}^{*}}{\rightarrow} \rho_{33(F L 1)}^{(2)}$ and $\rho_{00}^{(0)} \stackrel{G_{2}}{\rightarrow} \rho_{20}^{(1)} \stackrel{G_{2}^{*}}{\rightarrow} \rho_{22(F L 2)}^{(2)}$ shown in Fig. 1(c). The diagonal density matrix elements are $\rho_{33(F L 1)}^{(2)}=\frac{-G_{1}^{2}}{\left(\Gamma_{30}+i \Delta_{1}\right) \Gamma_{33}}$ and $\rho_{22(F L 2)}^{(2)}=\frac{-G_{2}^{2}}{\left(\Gamma_{20}+i \Delta_{2}\right) \Gamma_{22}}$. When $E_{1}$ and $E_{1}$ are on, the second-order fluorescence with dressing fields $E_{1}$ and $E_{1}$ are rewritten as follows:

$$
\rho_{33 D}^{(2)}=\frac{-G_{1}^{2}}{\left(\Gamma_{30}+i \Delta_{1}+\frac{G_{2}^{2}}{\Gamma_{32}+i\left(\Delta_{1}-\Delta_{2}\right)}\right) \Gamma_{33}}, \quad \rho_{22 D}^{(2)}=\frac{-G_{2}^{2}}{\left(\Gamma_{20}+i \Delta_{2}+\frac{G_{1}^{2}}{\Gamma_{23}+i\left(\Delta_{2}-\Delta_{1}\right)}\right) \Gamma_{22}} .
$$

Then, the fourth-order fluorescence is generated through the perturbation chains $\rho_{00}^{(0)} \stackrel{G_{2}}{\rightarrow} \rho_{20}^{(1)} \stackrel{G_{2}^{*}}{\rightarrow} \rho_{00}^{(2)} \stackrel{G_{1}}{\rightarrow} \rho_{30}^{(3)} \stackrel{G_{1}^{*}}{\rightarrow} \rho_{33(F L 3)}^{(4)}$ and $\rho_{00}^{(0)} \stackrel{G_{1}}{\rightarrow} \rho_{30}^{(1)} \stackrel{G_{1}^{*}}{\rightarrow} \rho_{00}^{(2)} \stackrel{G_{2}}{\rightarrow} \rho_{20}^{(3)} \stackrel{G_{2}^{*}}{\rightarrow} \rho_{22(F L 4)}^{(4)}$.

The diagonal density matrix element and given as follows:

$$
\rho_{33(F L 3)}^{(4)}=\frac{G_{1}^{2} G_{2}^{2}}{\left(\Gamma_{20}+i \Delta_{2}\right) \Gamma_{00}\left(\Gamma_{30}+i \Delta_{1}\right) \Gamma_{33}}, \quad \rho_{22(F L 4)}^{(4)}=\frac{G_{2}^{2} G_{1}^{2}}{\left(\Gamma_{30}+i \Delta_{1}\right) \Gamma_{00}\left(\Gamma_{20}+i \Delta_{2}\right) \Gamma_{22}}
$$

The fluorescence propagation equation is $I=I_{F L}-I_{A}$, where $I_{F L}=C N_{F L}^{2} \mu^{2} \int_{-\infty}^{+\infty}\left(e^{-(v / u)^{2}}\left|\rho^{(4)}(v)\right|^{2} / u \sqrt{\pi}\right) d v$ is the total intensity of the generated fluorescence signal. $\rho^{(4)}(\nu)$ is the density-matrix element of the fluorescence signal including pure fluorescence and multi-order fluorescence signals. $I_{A}$ is the absorption of the fluorescence signals in the medium and may be written as $I_{A}=I_{F L}\left(1-e^{-\alpha L}\right)=C N_{F L}^{2} \mu^{2} \int_{-\infty}^{+\infty}\left(e^{-(v / u)^{2}} K \operatorname{Im}[F L(v)] / u \sqrt{\pi}\right) d v$. Where $\alpha$ is the absorption co-efficient. $C$ is a constant. $K=I_{0} L k_{1} / C N_{F L} \hbar \varepsilon_{0} . F=\hbar \varepsilon_{0} \chi / N_{F L} \mu^{2}$ is the effective atom number. $\mu$ is the dipole moment. $v$ is the velocity of the atom due to Doppler effect. $u$ is the most probable velocity. For the coupling field fluorescence, the intensities of fluorescence are proportional to the $\rho_{33(F L)}^{(4)}$ and $\rho_{22(F L)}^{(4)}$, where the brackets express the time average $\left\langle I_{i}(t)\right\rangle=\int_{t}^{t+T} I_{i}(t) / T,\left\langle I_{i}\right\rangle$ is the average intensity of each laser beam and $I_{i}(t)$ gives the intensity versus time. $T$ is the time of integration. $I_{i}(t) \approx \Omega_{i}^{2}-2 \Omega_{i} \eta_{i} L \operatorname{Im}\left[\rho_{31 i}^{(1)}(t)\right]$ and $I_{j}(t) \approx \Omega_{j}^{2}-2 \Omega_{j} \eta_{j} L \operatorname{Im}\left[\rho_{32 j}^{(1)}(t)\right]$ are the intensities in this process. The correlations between the fluorescence $I_{m}$ and $I_{n}$ are given by the $G^{(2)}(\tau)$, which is a function between time delay $\tau$ and the intensities ${ }^{24}$ : 
(a)

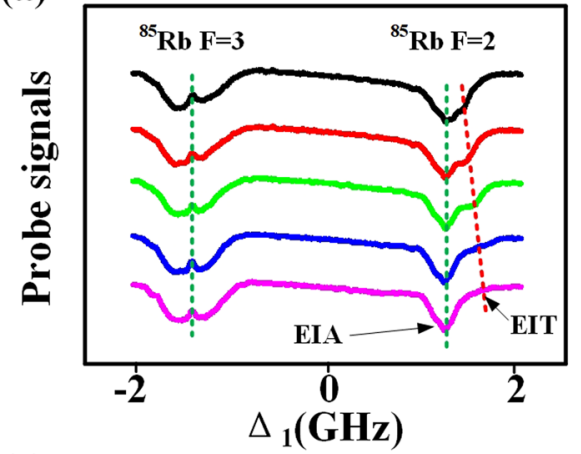

(c)

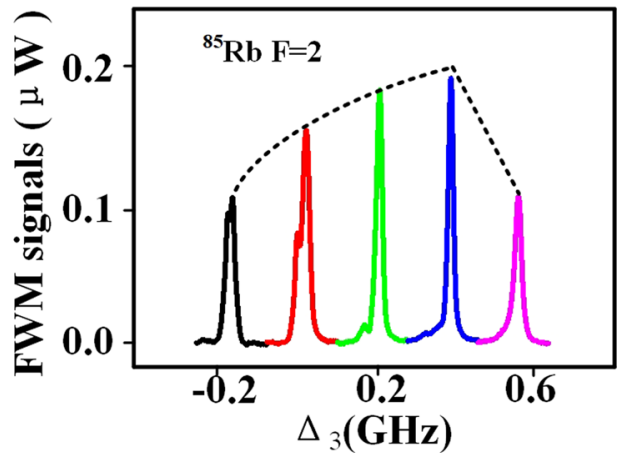

(b)

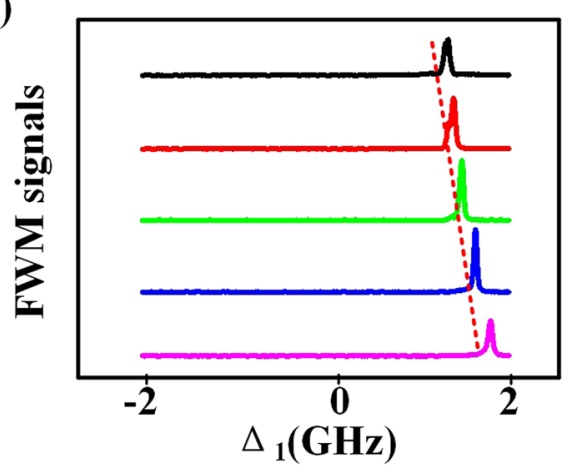

(d)

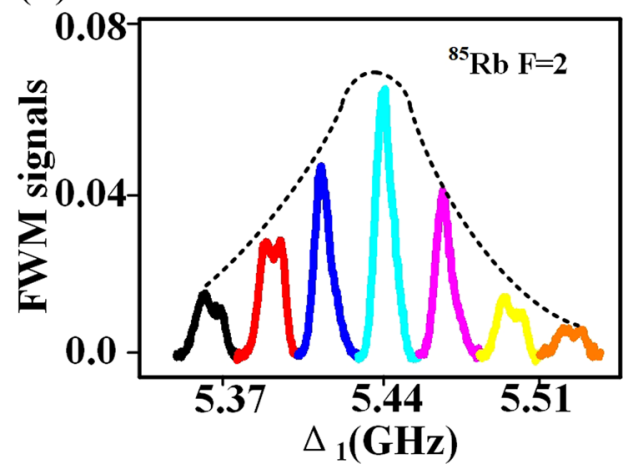

Figure 2. (a) By fixing the wavelength of $E_{2}$ at $794.981 \mathrm{~nm}$, the probe transmission spectra versus $\Delta_{1}$ which the five curves from top to bottom are obtained with increased $\Delta_{3}$. The red and green dotted lines are the fit lines of the EIT and EIA. (b) Measured FWM signals versus $\Delta_{1}$ at discrete $\Delta_{3}$ correspondings to (a). (c) The FWM signals $E_{F 1}$ with increased $\Delta_{3}$ from left to right corresponding to (b). The black dotted line is the fit line of the FWM signals. (d) The FWM signals $E_{F 1}$ with increased $\Delta_{1}$.

$$
\begin{aligned}
G^{(2)}(\tau) & =\frac{\left\langle I_{m}(t) I_{n}(t+\tau)\right\rangle}{\sqrt{\left\langle\left[I_{m}(t)\right]^{2}\right\rangle\left\langle\left[I_{n}(t+\tau)\right]^{2}\right\rangle}} \\
& =\frac{\left\langle\left\{\Omega_{m}^{2}-2 \Omega_{m} \eta_{m} L \operatorname{Im}\left[\rho_{m}^{(1)}(t)\right]\right\}\left\{\Omega_{n}^{2}-2 \Omega_{n} \eta_{n} L \operatorname{Im}\left[\rho_{n}^{(1)}(t+\tau)\right]\right\}\right\rangle}{\sqrt{\left\langle\left\{\operatorname{Im}\left[\rho_{m}^{(1)}(t)\right]\right\}^{2}\right\rangle\left\langle\left\{\operatorname{Im}\left[\rho_{n}^{(1)}(t+\tau)\right]\right\}^{2}\right\rangle}} \\
& \propto 1+\operatorname{sinc}^{2}\left(\frac{\Delta \omega \tau}{2 \pi}\right)
\end{aligned}
$$

\section{Results and Discussion}

First, the wavelength of the strong coupling laser $E_{2}$ was fixed at $794.981 \mathrm{~nm}$, which connects the $|1\rangle\left(5 S_{1 / 2}, \mathrm{~F}=3\right)$ and $|2\rangle\left(5 P_{1 / 2}\right)$ transition of the ${ }^{85} \mathrm{Rb} \mathrm{D} 1$ line in Fig. $1(\mathrm{a})$. The frequency of the pump laser $E_{1}$ was monitored and scanned over the entire range of ground and excited states. By changing the detuning $\Delta_{3}$ from -0.2 to $0.6 \mathrm{GHz}$, we observed the positions of standard $\Lambda$-EIT window $\left(E_{1}\right.$ and $E_{3}$ satisfying $\left.\Delta_{1}-\Delta_{3}=0\right)$ on the typical probe transmission spectrum in Fig. 2(a) and the intensity variation of FWM signals detected by the APD1 in Fig. 2(b). While the $\Lambda$-EIT windows are moved in the positive direction along $\Delta_{1}$-axis by the increasing $\Delta_{3}$. Five sharp peaks of $E_{F 1}$ on the FWM spectrum are observed falling into the $\Lambda$-EIT windows corresponding to Fig. 2(a). The phenomenon indicates that the primary cause of $E_{F 1}$ switches is atomic coherence. Additionally, we use the saturated absorption technique and EIT peaks to calibrate the positions of the coupling and pump beams on the probe transmission spectrum. The windows can be identified by fixing the different incident beams and increasing detuning of the other laser beams. The intensity of the FWM signals can be controlled easily by adjusting the detuning of the $E_{3}$ in Fig. 2(c). The maximum enhancement of the $E_{F 1}$ signal is approximately $0.2 \mu \mathrm{W}$ when $\Delta_{3}=0.4 \mathrm{GHz}$ is satisfied.

In the following, we show the effect of each window by scanning the detuning $\Delta_{1}$ at different detuning $\Delta_{2}$. This method is very convenient for the observation of suppression and enhancement. The measured probe curves versus $\Delta_{1}$ shown in Fig. 3(a), where the seven curves from top to bottom are obtained with increased $\Delta_{2}$. Corresponding to Fig. 2(a), the measured FWM curves versus $\Delta_{1}$ at discrete $\Delta_{2}$ are shown in Fig. 3(c). We observed the FWM signal $E_{F 2}$ and $E_{F 3}$ from the "double $\Lambda$ " four-level atomic systems with fixed beam $E_{3}$ $(780.237 \mathrm{~nm})$. The $\Lambda$-EIT windows formed by $E_{1}$ and $E_{3}$ in the dip of $\mathrm{F}=3$ does not move with the increase of $\Delta_{2}$ in Fig. 3(a). While the new type EIT windows are moved in the negative direction along $\Delta_{1}$-axis. Since, the detuning of probe beams is fixed, the peaks of $E_{F 2}$ and $E_{F 3}$ are also fixed in Fig. 3(c). Additionally, we use the 
(a)

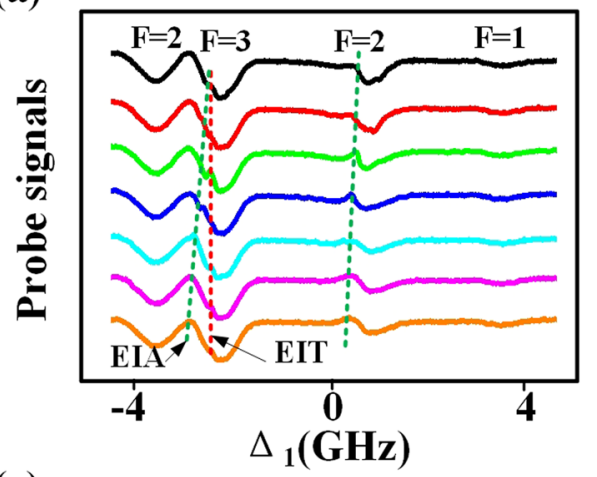

(c)

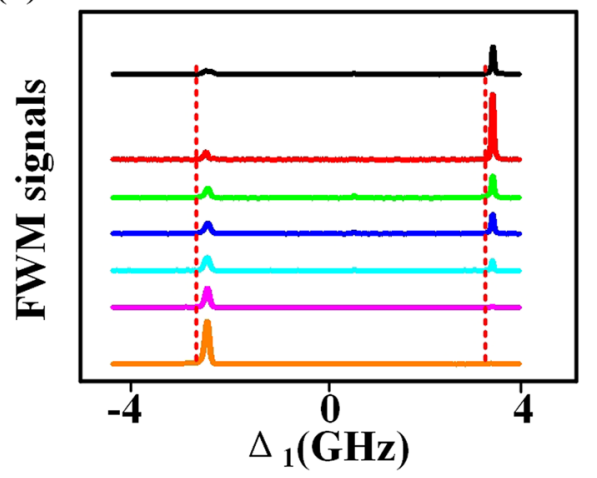

(b)

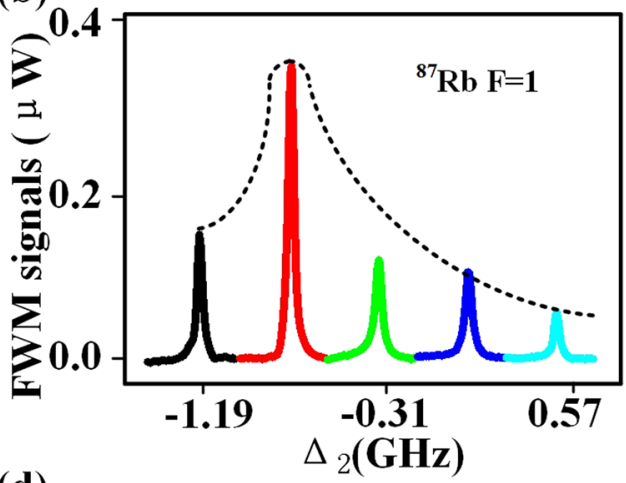

(d)

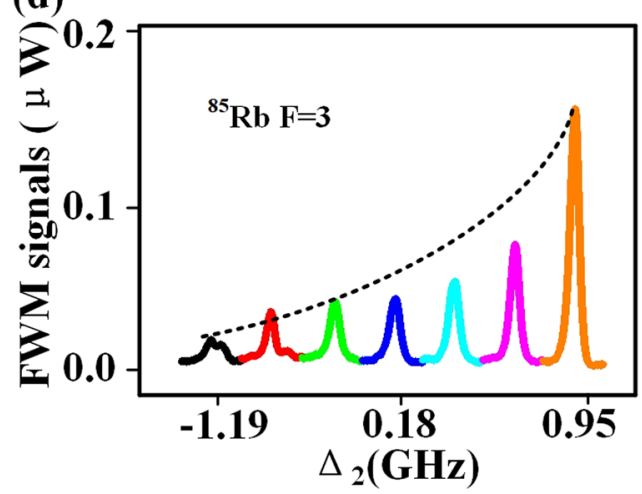

Figure 3. (a) By fixing the wavelength of $E_{3}$ at $780.237 \mathrm{~nm}$, the probe transmission spectrum versus $\Delta_{1}$ which the seven curves from top to bottom are obtained with increasing $\Delta_{2}$. The red and green dotted lines are the fit lines of the EIT and EIA. (b) The FWM signals $E_{F 2}$ of ${ }^{87} \mathrm{Rb} \mathrm{F}=1$ with increased $\Delta_{2}$ from left to right. The black dotted line is the fit line of the FWM signals. (c) Measured FWM signals versus $\Delta_{1}$ at discrete $\Delta_{2}$ corresponding to (a). (d) The FWM signals $E_{F 3}$ of ${ }^{85} \mathrm{Rb} \mathrm{F}=3$ with increased $\Delta_{2}$ from left to right.

saturated absorption technique and EIT peaks to calibrate the positions of the coupling and pump beams on the probe transmission spectrum. Similarly, the windows can also be identified by fixing the different incident beams and increasing detuning of the other laser. The intensity of the FWM signals can be controlled easily by adjusting the detuning of the coupling beam in Fig. $3(\mathrm{~b}, \mathrm{~d})$. The maximum enhancement of the $E_{F 3}$ signal is approximately $0.35 \mu \mathrm{W}$ when $\Delta_{2}=-0.75 \mathrm{GHz}$ is satisfied. When $\Delta_{2}$ changes from -1.19 to $0.95 \mathrm{GHz}$ discretely, the $E_{F 2}$ signal increased to $0.15 \mu \mathrm{W}$. Moreover, the linewidth of each window is narrow, the induced suppression (or enhancement) of $E_{F 1}$ is very sensitive to the relative position between each other.

At last, we block the injection laser $E_{3}$ and fix the wavelength of $E_{1}$ and $E_{2}$ at 780.2396 and $794.9828 \mathrm{~nm}$ respectively. Then adjust the detectors into two SPCMs. The Stokes and anti-Stokes paired photons generated from the SP-FWM nonlinear process simultaneously, which propagate in opposite direction in Fig. 1(a) in the hot atomic ensemble. The Stokes photons usually propagate through the atomic transitions with the speed of light in vacuum $c$. And the anti-Stokes photons propagate through a coherent $\Lambda$-EIT window which determines the paired photons correlation time and waveforms. Simultaneously, the biphoton coincidence counts are detected by two independent SPCMs and recorded by a time-to-digit converter with a temporal bin width of $0.0244 \mathrm{~ns}$. The measurement results of the signals ineluctably include the other three parts: the correlated fourth-order fluorescence, linear Rayleigh scattering and uncorrelated second-fluorescence.

We observed the FWM signal $E_{F 2}$ and $E_{F 3}$ from the "double $\Lambda$ " four-level atomic systems with fixed beam $E_{3}$ $(780.237 \mathrm{~nm})$. The $\Lambda$-EIT windows formed by $E_{1}$ and $E_{3}$ in the dip of $\mathrm{F}=3$ does not move by increasing the $\Delta_{2}$ in Fig. 3(a). While the new type EIT windows are moved in the negative direction along $\Delta_{1}$-axis. Since the detuning of probe beams is fixed, the peaks of $E_{F 2}$ and $E_{F 3}$ are also fixed in Fig. 3(c). Additionally, we use the saturated absorption technique and EIT peaks to calibrate the positions of the coupling and pump beams on the probe transmission spectrum. Similarly, the windows can also be identified by fixing the different incident beams and increasing detuning of the other laser. The intensity of the FWM signals can be controlled easily by adjusting the detuning of the coupling beam in Fig. 3. The maximum enhancement of the $E_{F 3}$ signal is approximately $0.35 \mu \mathrm{W}$ when $\Delta_{2}=-0.75 \mathrm{GHz}$ is satisfied. When $\Delta_{2}$ changes from -1.19 to $0.95 \mathrm{GHz}$ discretely, the $E_{F 2}$ signal monotonically increased to $0.15 \mu \mathrm{W}$. Moreover, the linewidth of each window is narrow, the induced suppression (or enhancement) of $E_{F 1}$ is very sensitive to the relative position between each other.

Physically, this waveform can be explained as follows: we calculate the coherence time of the different paired photons to distinguish their contribution to the result of coincidence counts. The effective dephasing rate of $E_{S}$ and $E_{a S}$ is $\Gamma_{e}=\left(\Gamma_{10}+\Gamma_{30}\right) / 2$, thus their coherence time is 116.3 ns shown in the Eq. 3 , which agrees well with the experiment result in Fig. 4. While the coherence time of fourth-order fluorescence is 18.2 ns shown in the Eq. 6. 


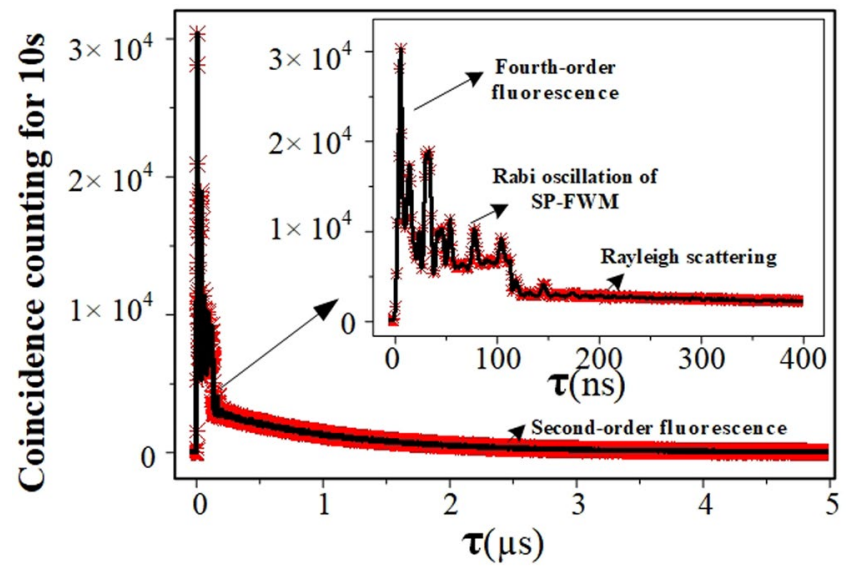

Figure 4. By fixing the wavelength of $E_{1}$ and $E_{2}$ at $780.2396 \mathrm{~nm}$ and $794.9828 \mathrm{~nm}$ respectively, biphoton coincidence counts as function of relative time delay $\tau$ between paired Stokes and anti-Stokes and multi-order fluorescence photons collected over $10 \mathrm{~s}$ with $0.0244 \mathrm{~ns}$ bin width.

So it is easy to distinguish these two paired photons. The background nonzero floor is a result of accidental coincidence between the on-resonance Rayleigh scattering, the second-order fluorescence and uncorrelated Stokes and anti-Stokes photons from different pairs. These photons have the same polarization and central frequency as the Stokes and anti-Stokes photons. So they cannot be filtered away by the polarization and frequency filters.

\section{Conclusion}

In conclusion, we used hot atomic-gas media to generate non-classical light through the SP-FWM process in four-energy level system, especially focusing on biphoton generation. Our work shows that the pump and coupling field corporately determine the EIT. The EIT dephasing rate and loss determined the biphoton correlation time and waveforms. Meanwhile, we also observed the biphoton Rabi oscillation of SP-FWM with correlation time $116.3 \mathrm{~ns}$. The background was attributed to linear Rayleigh scattering and uncorrelated second-fluorescence. We experimented with the method of using hot atoms to analyze and suppress the influence of the noise term, that is, the uncorrelated terms. This work pave the way for finding suitable modules for quantum communication.

\section{Methods}

Experimental setup. The experiments are carried out in a "double- $\Lambda$ " four-level $\left(|0\rangle\left(5 S_{1 / 2}, \mathrm{~F}=2\right),|1\rangle\left(5 S_{1 / 2}\right.\right.$, $\mathrm{F}=3),|2\rangle\left(5 P_{1 / 2}\right)$ and $\left.|3\rangle\left(5 P_{3 / 2}\right)\right)$ atomic systems of ${ }^{85} \mathrm{Rb}$ shown in Fig. 1 . The strong pump laser beam $E_{1}$, the coupling beam $E_{2}$ and the weak probe laser beam $E_{3}$ are emitted by the LD1-3 with the diameter $0.2 \mathrm{~mm}$, respectively. The angle between the beams $E_{1}$ and $E_{3}$ is $0.26^{\circ}$. A thermal temperature-stabilized rubidium vapor cell with length of $L=5.5 \mathrm{~cm}$ is heated up $55^{\circ} \mathrm{C}$ in center of this experiment setup and the atom density is about $2.5 \times 10^{11} \mathrm{~cm}^{-3}$ in order to have enough atoms in the cavity to enhance the strength of atom-cavity coupling. Blocking the injection laser $E_{3}$ and using two detectors (avalanche diode, PerkinElmer SPCM-AQR-15-FC, 50\% efficiency, maximum dark count rate of 50/s)), the biphoton coincidence counts with fluorescence signals can be detected.

Third-order density matrix elements of PA-FWM. When $E_{3}$ is injected into the Stokes port of SP-FWM process in stokes channel, the generated $E_{S}$ and $E_{a S}$ signals in PA-FWM can be described by the third-order density matrix elements, which could be obtained by the perturbation chains $\rho_{11}^{(0)} \stackrel{\omega_{1}}{\rightarrow} \rho_{31}^{(1)} \stackrel{-\omega_{a S}}{\longrightarrow} \rho_{01}^{(2)} \stackrel{\omega_{2}}{\rightarrow} \rho_{21(S)}^{(3)}$ and $\rho_{00}^{(0)} \stackrel{\omega_{2}}{\rightarrow} \rho_{20}^{(1)} \stackrel{-\omega_{3}}{\longrightarrow} \rho_{10}^{(2)} \stackrel{\omega_{1}}{\rightarrow} \rho_{30(a S)}^{(3)}:$

$$
\rho_{21(S)}^{(3)}=-i G_{1} G_{a S} G_{2} / d_{11} d_{31} d_{01}, \quad \rho_{20(a S)}^{(3)}=-i G_{2} G_{3} G_{1} / d_{20} d_{10} d_{30} .
$$

where $G_{i}=\mu_{i j} E_{i} / \hbar(i, j=1,2,3, a S)$ is the Rabi frequency between levels $|i\rangle \leftrightarrow|j\rangle$, and $\mu_{i j}$ is the dipole momentum; $d_{30}=\Gamma_{30}+i\left(\Delta_{2}-\Delta_{3}+\Delta_{1}\right), \quad d_{11}=\Gamma_{11}+i \Delta_{1}, \quad d_{31}=\Gamma_{31}, \quad d_{01}=\Gamma_{01}+i \Delta_{2}, \quad d_{20}=\Gamma_{20}+i \Delta_{2}$, $d_{10}=\Gamma_{10}+i\left(\Delta_{2}-\Delta_{3}\right), \Gamma_{i j}=\left(\Gamma_{i}+\Gamma_{j}\right) / 2$ is the decoherence rate between $|i\rangle$ and $|j\rangle ; \Delta_{i}=\Omega_{i}-\omega_{i}$ is detuning defined as the difference between the resonant transition frequency $\Omega_{i}$ and the laser frequency $\omega_{i}$ of $E_{i}$.

The photon numbers of the output Stokes and anti-Stokes fields of optical parametric amplification can be described as:

$$
\left\langle N_{a}\right\rangle=\left\langle\hat{\mathrm{a}}_{\text {out }}^{\dagger} \hat{\mathrm{a}}_{\text {out }}\right\rangle=g\left\langle\hat{\mathrm{a}}_{\text {in }}^{\dagger} \hat{\mathrm{a}}_{\text {in }}\right\rangle+(g-1), \quad\left\langle N_{b}\right\rangle=\left\langle\hat{b}_{\text {out }}^{\dagger} \hat{b}_{\text {out }}\right\rangle=(g-1)\left\langle\hat{\mathrm{a}}_{\text {in }}^{\dagger} \hat{\mathrm{a}}_{\text {in }}\right\rangle+g .
$$

where $g=\left\{\cos \left[2 t \sqrt{A B} \sin \left(\phi_{1}+\phi_{2}\right) / 2\right]+\cosh \left[2 t \sqrt{A B} \cos \left(\phi_{1}+\phi_{2}\right) / 2\right]\right\} / 2$ is the dressed SP-FWM gain with the modulus $A$ and $B$ (phase angles $\varphi_{1}$ and $\varphi_{2}$ ) defined in $\rho_{21(S)}^{(3)}=A e^{i \phi_{1}}$ and $\rho_{20(a S)}^{(3)}=B e^{i \phi_{2}}$, respectively. 


\section{Data availability}

The data is available from the corresponding author on reasonable request.

Received: 10 July 2019; Accepted: 26 November 2019;

Published online: 27 December 2019

\section{References}

1. Horodecki, R., Horodecki, P., Horodecki, M. \& Horodecki, K. Quantum entanglement. Rev. Mod. Phys. 81, 865 (2009).

2. Kimble, H. J. The quantum internet. Nature 453, 1023-30 (2008).

3. Bennett, C. H. \& DiVincenzo, D. P. Quantum information and computation. Nature 404, 247 (2000).

4. Lugiato, L., Gatti, A. \& Brambilla, E. Quantum imaging. J. Opt. B: Quantum semiclassical optics 4, S176 (2002).

5. Brida, G., Genovese, M. \& Berchera, I. R. Experimental realization of sub-shot-noise quantum imaging. Nat. Photonics 4, 227 (2010).

6. Migdall, A., Datla, R., Sergienko, A., Orszak, J. S. \& Shih, Y. H. Measuring absolute infrared spectral radiance with correlated visible photons: technique verification and measurement uncertainty. Appl. Optics 37, 3455-3463 (1998).

7. Walther, P. et al. De broglie wavelength of a non-local four-photon state. Nature 429, 158 (2004).

8. Kwiat, P. G. et al. New high-intensity source of polarization-entangled photon pairs. Phys. Rev. Lett. 75, 4337 (1995).

9. Rubin, M. H., Klyshko, D. N., Shih, Y. \& Sergienko, A. Theory of two-photon entanglement in type-ii optical parametric downconversion. Phys. Rev. A 50, 5122 (1994).

10. Halder, M. et al. Entangling independent photons by time measurement. Nat. Phys. 3, 692 (2007).

11. Du, S., Kolchin, P., Belthangady, C., Yin, G. \& Harris, S. Subnatural linewidth biphotons with controllable temporal length. Phys. Rev. Lett. 100, 183603 (2008).

12. Zhao, L. et al. Photon pairs with coherence time exceeding $1 \mu$ s. Optica 1, 84-88 (2014).

13. Han, Z., Qian, P., Zhou, L., Chen, J. \& Zhang, W. Coherence time limit of the biphotons generated in a dense cold atom cloud. Sci. Reports 5, 9126 (2015).

14. Zhao, L. et al. Shaping the biphoton temporal waveform with spatial light modulation. Phys. Rev. Lett. 115, 193601 (2015).

15. Fang, Y. \& Jing, J. Quantum squeezing and entanglement from a two-mode phase-sensitive amplifier via four-wave mixing in rubidium vapor. New J. Phys. 17, 023027 (2015).

16. Yan, H. et al. Generation of narrow-band hyperentangled nondegenerate paired photons. Phys. Rev. Lett. 106, 033601 (2011).

17. Zheng, H. et al. Parametric amplification and cascaded-nonlinearity processes in common atomic system. Sci. Reports 3, 1885 (2013).

18. Jeong, H., Du, S. \& Kim, N. Y. Proposed narrowband biphoton generation from an ensemble of solid-state quantum emitters. J. Opt. Soc. Am. B 36, 646-651 (2019).

19. Zhao, L., Su, Y. \& Du, S. Narrowband biphoton generation in the group delay regime. Phys. Rev. A 93, 033815 (2016).

20. Zhu, L., Guo, X., Shu, C., Jeong, H. \& Du, S. Bright narrowband biphoton generation from a hot rubidium atomic vapour cell. Appl. Phys. Lett. 110, 161101 (2017).

21. Zhang, Z. et al. Dressed gain from the parametrically amplified four-wave mixing process in an atomic vapor. Sci. Rep. 5, 15058 (2015).

22. Wen, J. \& Rubin, M. H. Transverse effects in paired-photon generation via an electromagnetically induced transparency medium. ii. beyond perturbation theory. Phys. Rev. A 74, 023809 (2006).

23. Du, S., Wen, J. \& Rubin, M. H. Narrowband biphoton generation near atomic resonance. J. Opt. Soc. Am. B 25, C98-C108 (2008).

24. Tang, H. et al. Investigation of multi-bunching by generating multi-order fluorescence of nv center in diamond. Phys. Chem. Chem. Phys. 20, 5721-5725 (2018).

\section{Acknowledgements}

National Key R\&D Program of China (2017YFA0303700, 2018YFA0307500); National Natural Science Foundation of China $(61605154,11604256,11804267)$.

\section{Author contributions}

Y.L. wrote the main manuscript and contributed to experimental work. Y.P.Z. provided the idea. K.K.L., S.Q.Z., H.R.F. and W.L. contributed to the presentation and execution of the theoretical work. All authors discussed the results and contributed to the writing of the manuscript.

\section{Competing interests}

The authors declare no competing interests.

\section{Additional information}

Correspondence and requests for materials should be addressed to Y.Z.

Reprints and permissions information is available at www.nature.com/reprints.

Publisher's note Springer Nature remains neutral with regard to jurisdictional claims in published maps and institutional affiliations.

(c) (i) Open Access This article is licensed under a Creative Commons Attribution 4.0 International

License, which permits use, sharing, adaptation, distribution and reproduction in any medium or format, as long as you give appropriate credit to the original author(s) and the source, provide a link to the Creative Commons license, and indicate if changes were made. The images or other third party material in this article are included in the article's Creative Commons license, unless indicated otherwise in a credit line to the material. If material is not included in the article's Creative Commons license and your intended use is not permitted by statutory regulation or exceeds the permitted use, you will need to obtain permission directly from the copyright holder. To view a copy of this license, visit http://creativecommons.org/licenses/by/4.0/.

(C) The Author(s) 2019 